EPJ Web of Conferences 75, 03002 (2014)

DOI: 10.1051/epjconf/20147503002

(C) Owned by the authors, published by EDP Sciences, 2014

\title{
Modelling of Packed Co Nanorods for Hard Magnetic Applications
}

\author{
P. Toson ${ }^{1, \mathrm{a}}$, W. Wallisch ${ }^{1}$, A. Asali ${ }^{1}$, J. Fidler $^{1}$ \\ ${ }^{1}$ Institute of Solid State Physics, Vienna University of Technology, 1040 Vienna, Austria
}

\begin{abstract}
We present a numerical algorithm based on the bullet physics library to generate densely packed $(39 \%-41 \%)$ structures of high-aspect-ratio nanorods for finite element micromagnetic simulations. The coercivities $\mu_{0} \mathrm{H}_{\mathrm{c}}$ of the corresponding Cobalt nanorod structures vary between $0.50 \mathrm{~T}$ and $0.67 \mathrm{~T}$, depending on the overall orientation of nanorods, which is in good agreement with experimental results. The simulations make it possible to estimate the coercivity loss due to incoherent reversal processes $(27 \%)$ as well as the gain due to shape anisotropy (59\%). Our calculations show permanent magnets consisting of packed Co nanorods with an energy density product $(\mathrm{BH})_{\max }$ of $83 \mathrm{~kJ} / \mathrm{m}^{3}$. We estimate that this value can be increased to $103 \mathrm{~kJ} / \mathrm{m}^{3}$ by increasing the packing density from $40 \%$ to $45 \%$. Another way to optimize (BH) $\max$ is the usage of novel materials. By varying the anisotropy constant $\mathrm{K}_{1}$ and the saturation polarisation $\mathrm{J}_{\mathrm{S}}$ we found lower limits for these parameters to reach a certain energy density product. To increase $(\mathrm{BH})_{\max }$ to $160 \mathrm{~kJ} / \mathrm{m}^{3}, \mathrm{~K}_{1}$ and $\mathrm{J}_{\mathrm{S}}$ have to be in the order of $450 \mathrm{~kJ} / \mathrm{m}^{3}$ and $2.25 \mathrm{~T}$, respectively. The thermal stability of this approach was verified by elastic band calculations. Cobalt nanorods with a diameter of $10 \mathrm{~nm}$ and a height of $50 \mathrm{~nm}$ are thermally stable at room temperature, but problematic at $900 \mathrm{~K}$. Doubling the nanorods' height to $100 \mathrm{~nm}$ increases that limit considerably.
\end{abstract}

\section{Introduction}

High magnetocrystalline and shape anisotropy are preconditions for achieving hard magnetic properties. A densely packed structure of high-aspect-ratio (1:5 - 1:10) cobalt nanorods exploits both sources of anisotropy and experiments showed that these structures maintain high coercivity values at high temperatures, because the shape anisotropy is temperature-independent [1].
We present a method to generate realistically packed Co nanorods for finite element micromagnetic simulations. We examined the influence of shape anisotropy, incoherent reversal processes, alignment of nanorods and packing density on the magnetic properties.

Based on the results from packed models, we performed simulations on a single nanorod with varying material parameters (anisotropy constant $\mathrm{K}_{1}$ and saturation polarisation $\mathrm{J}_{\mathrm{s}}$ ) and estimated the energy
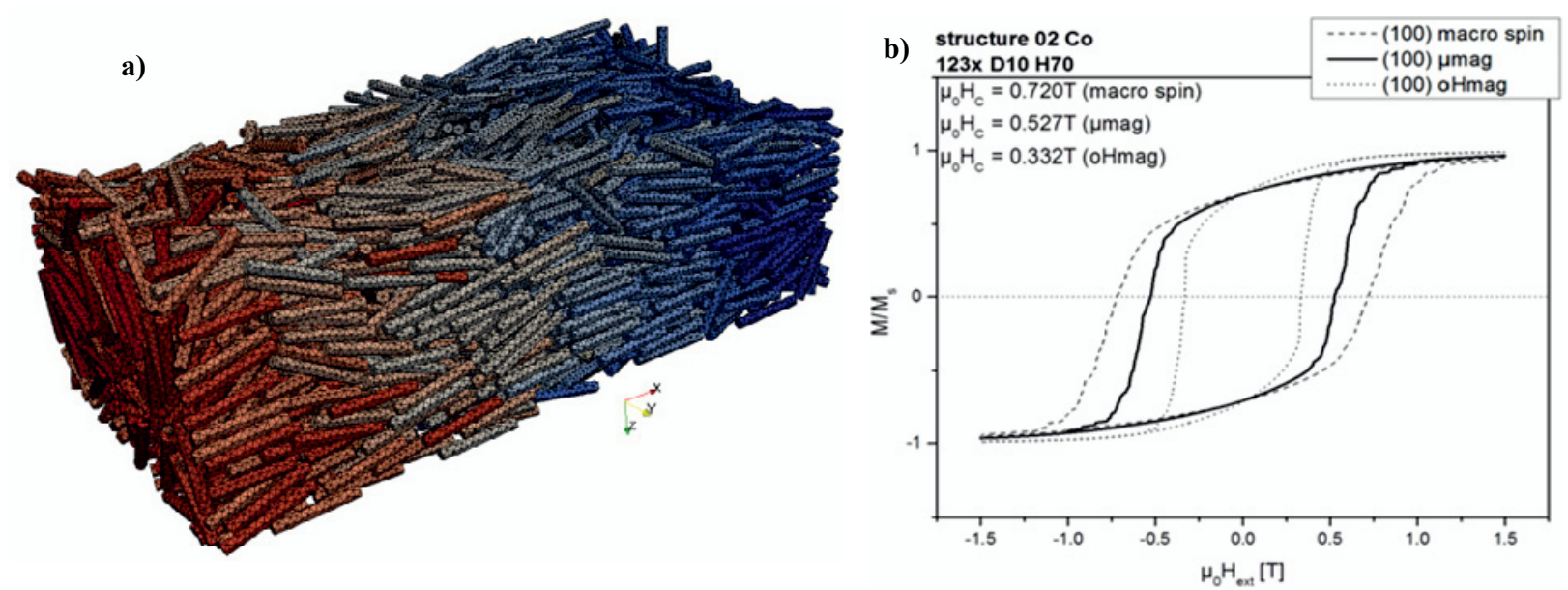

Fig 1. a) Finite element model of a Co structure with 3200 nanorods. b) Calculated hysteresis properties according to the three simulation methods: Full micromagnetics ( $\mu \mathrm{mag}$ ) give the most accurate results, macro spin assumes coherent reversal and oHmag takes only crystal anisotropy into account. 
density product $(\mathrm{BH})_{\max }$ of a packed structure to demonstrate the limits of packed nanorods for hard magnetic applications.

In addition, we performed energy barrier calculations to determine the thermal stability of nanorods which restricts the size of nanorods in high-temperature applications.

\section{Packed Nanorods}

We introduced an algorithm based on the bullet physics library [2] to generate realistically packed structures of nanorods. The nanorods are modelled as ideal, stiff cylinders and their motion is based on gravity and collision forces between nanorods and the walls as well as between nanorods themselves. To obtain nanorod structures with different misalignments, we introduced a tuneable torque on each nanorod which mimics the alignment of magnetic dipoles in a homogenous external field. The misalignment is measured by the standard deviation of angles $\phi$ between the external field (along the $\mathrm{x}$-axis) and the orientation of the nanorods, denoted as $\sigma_{\phi}$. Using 3200 nanorods with diameter $\mathrm{D}=10 \mathrm{~nm}$ and height $\mathrm{H}=70 \mathrm{~nm}$ we obtained structures with $\sigma_{\phi}$ between $10.0^{\circ}$ and $71.7^{\circ}$ and the packing density varies between $37 \%$ and $41 \%$. An example structure is shown in figure 1 a.

The magnetic properties of Co nanorod structures have been calculated by the finite element micromagnetic package FEMME which solves the Landau-LifshitzGilbert equations [3]. Because a full micromagnetic simulation ( $\mu \mathrm{mag}$ ) on a structure with 3200 nanorods is not feasible, we performed the calculations on a subset of 120-140 nanorods. However, the complete 3200 nanorod structure can be calculated by a macro spin calculation. Technically, only one magnetisation vector is used per nanorod in a macro spin calculation. The physical interpretation of this simplification is that we assume coherent reversal in each nanorod. Please note that this is still not Stoner-Wolfarth coherent reversal, because the stray field interaction is calculated numerically and fully taken into account. The macro spin method yields correct values for the remanence, but the coercivity field is overestimated. The difference between the macro spin and the $\mu$ mag coercivity can be interpreted as the loss due to incoherent reversal processes (about 27\% across all structures).

In addition to $\mu$ mag and macro spin calculations, we performed simulations without the demagnetising field (oHmag). The oHmag method yields correct values for the remanence as well, but the coercivity field is only based on magnetocrystalline anisotropy. The difference between the oHmag and the $\mu$ mag coercivity is the gain due to shape anisotropy or the loss due to stray field effects. The shape anisotropy increases the coercivity field by $59 \%$ in packed Co nanorod structures. Figure $1 \mathrm{~b}$ shows 3 hysteresis loops for the same structure calculated by the macro spin, $\mu$ mag and oHmag method. The results are in good agreement with experimental data [1].
Remanence and coercivity are decreasing with increasing misalignment $\sigma_{\phi}$. In our observed range of misalignment $\left(10.0^{\circ}-71.7^{\circ}\right)$ the remanence $B_{r}$ varies between $0.50 \mathrm{~T}$ and $0.67 \mathrm{~T}$ and the coercivity field $\mu_{0} \mathrm{H}_{\mathrm{c}}$ varies between $0.53 \mathrm{~T}$ and $0.61 \mathrm{~T}$ (see figure 2). The values for the remanence are corrected by the packing density, because we are interested in the macroscopic flux density and not in the flux density in the magnetic material alone. The coercivities in are calculated by the $\mu$ mag method (and are therefore only available for the small structures with 120-140 nanorods) and the remanence values are taken from macro spin calculations (including full structures as well as subsets).

The energy density product $(\mathrm{BH})_{\max }$ can be calculated directly from the hysteresis loop (see figure 3 ). Considering the packing density of $40 \%$, we obtain an energy density of $83 \mathrm{~kJ} / \mathrm{m}^{3}$. If it would be possible to increase the packing density to $45 \%$ the energy density would increase to $103 \mathrm{~kJ} / \mathrm{m}^{3}$ assuming that the overall shape of the hysteresis loop does not change.

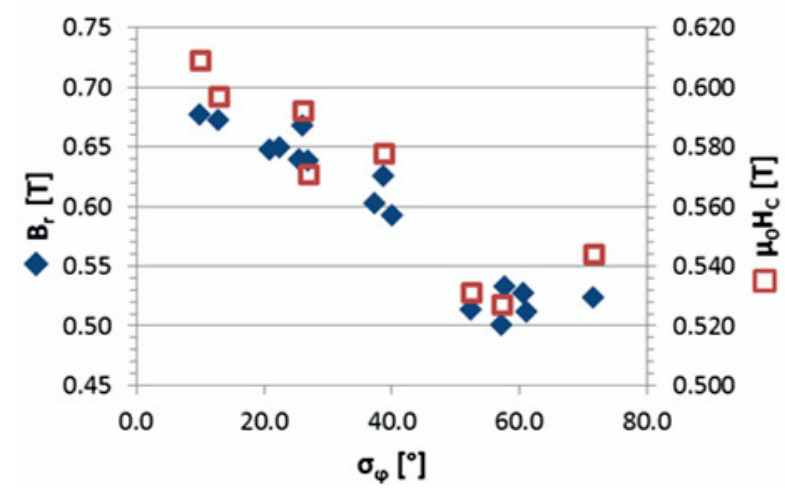

Fig 2. Dependence of remanence (diamonds) and coercivity fields (squares) on misalignment $\sigma_{\phi}$.

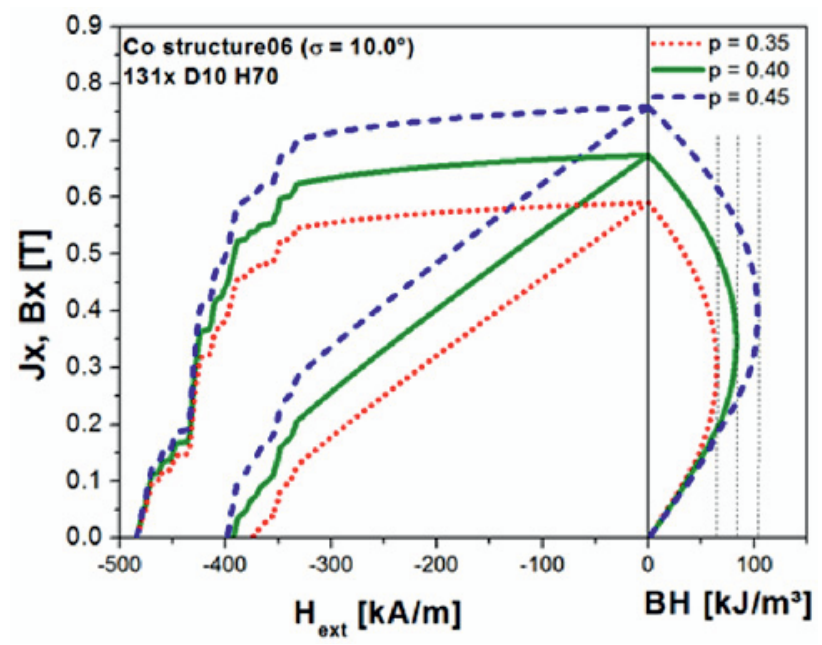

Fig 3. Calculation of the energy density product from the hysteresis loop of a packed Co nanorod structure. The full line uses the actual packing density of $40 \%$, dotted lines are approximations for a higher and lower packing density. 


\section{Variation of Material Parameters}

Another way to increase the energy density product is the usage of other (and possibly novel) materials. We varied the anisotropy constant $\mathrm{K}_{1}\left(45 \mathrm{k}-4.5 \mathrm{MJ} / \mathrm{m}^{3}\right)$ and the saturation polarisation $\mathrm{J}_{\mathrm{s}}(1.25 \mathrm{~T}-2.25 \mathrm{~T})$ to simulate new materials with uniaxial magnetocrystalline anisotropy and give lower limits for these parameters to obtain a certain energy density product.

The simulations for the parameter variation have been performed on a single D10H50 nanorod. Figure 4 shows the coercivity field as a function of $\mathrm{K}_{1}$ and $\mathrm{J}_{\mathrm{s}}$. The coercivity increases with increasing $\mathrm{K}_{1}$ and decreases with increasing $\mathrm{J}_{\mathrm{s}}$ for anisotropies above $120 \mathrm{~kJ} / \mathrm{m}^{3}$ due to stray field effects. Nanorods with a $\mathrm{K}_{1}$ below $120 \mathrm{~kJ} / \mathrm{m}^{3}$ profit from a higher saturation, because the higher stray field causes a higher shape anisotropy. It should be noted, that the exact break-even point depends on the shape of the nanorod. For D20H100 nanorods, this point is at $\mathrm{K}_{1}=80 \mathrm{~kJ} / \mathrm{m}^{3}$ (see figure $4 \mathrm{~b}$ ).

The energy density product can be again obtained from the hysteresis loop. To estimate the energy density of a packed model based on a single nanorod, we regard the packing density of $40 \%$ (which reduces the effective magnetic flux density) and assume that the coercivity loss of $60 \%$ due to the packing for Co nanorods stays the same across all material parameters.

Figure 5 summarizes the results for packed structures of D10H50 nanorods. The energy density product increases with $\mathrm{K}_{1}$ until the saturation polarisation limits the maximum energy density. Analytically, the energy density product of a broad $\left(\mu_{0} \mathrm{H}_{\mathrm{cJ}}>\mathrm{B}_{\mathrm{r}}\right)$ rectangular

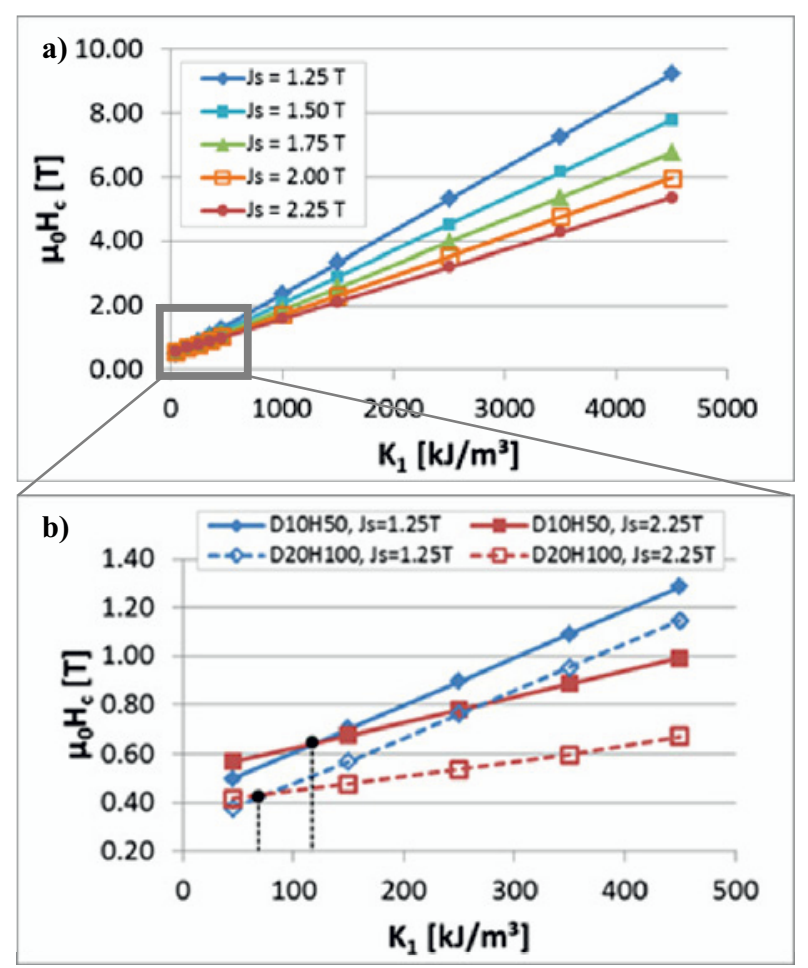

Fig 4. a) Influence of the anisotropy constant $K_{1}$ and the saturation polarisation $\mathrm{J}_{\mathrm{s}}$ on the coercivity field of a single D10H50 nanorod. b) shows the coercivities at lower K1 for D10H50 and D20H100 nanorods hysteresis loop can be calculated with:

$$
(B H)_{\max }=B_{r}^{2} / 4 \mu_{0}
$$

If we assume

$B_{r}=p J_{s}$

with packing density $p$, the analytical estimation agrees well with the upper limit of $(\mathrm{BH})_{\max }$ obtained from the simulation results. Table 1 lists lower limits for $\mathrm{K}_{1}$ and $\mathrm{J}_{\mathrm{s}}$ to achieve certain energy density product values. Please note that due to the nature of the estimation, these are necessary but not sufficient conditions.

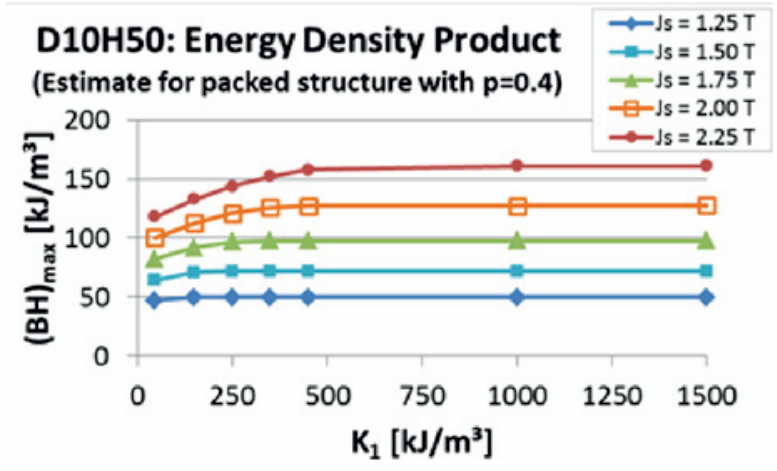

Fig 5. Estimation of the energy density product of a packed nanorod structure for various material parameters.

Table 1. Necessary lower limits for material parameters to achieve certain energy density products of a packed nanorod structure with a packing density of $40 \%$.

\begin{tabular}{|r|r|r|}
\hline \multicolumn{1}{|c|}{$(\mathbf{B H})_{\max }$} & \multicolumn{1}{c|}{$\boldsymbol{\text { min. } \mathbf { J } _ { \mathbf { s } }}$} & \multicolumn{1}{c|}{$\boldsymbol{\operatorname { m i n }}$. $\mathbf{K}_{\mathbf{1}}$} \\
\hline $70 \mathrm{~kJ} / \mathrm{m}^{3}$ & $1.50 \mathrm{~T}$ & $150 \mathrm{~kJ} / \mathrm{m}^{3}$ \\
\hline $90 \mathrm{~kJ} / \mathrm{m}^{3}$ & $1.75 \mathrm{~T}$ & $250 \mathrm{~kJ} / \mathrm{m}^{3}$ \\
\hline $120 \mathrm{~kJ} / \mathrm{m}^{3}$ & $2.00 \mathrm{~T}$ & $350 \mathrm{~kJ} / \mathrm{m}^{3}$ \\
\hline $160 \mathrm{~kJ} / \mathrm{m}^{3}$ & $2.25 \mathrm{~T}$ & $450 \mathrm{~kJ} / \mathrm{m}^{3}$ \\
\hline
\end{tabular}

\section{Thermal Stability}

For permanent magnets consisting of nanorods, thermal stability has to be considered. Analytically, the energy barrier to switch a nanorod can be calculated with:

$\Delta E=K_{I} V=k_{B} T$

If the energy barrier is higher than $100 \mathrm{k}_{\mathrm{B}} \mathrm{T}$, the magnet can be considered thermally stable.

In addition to the analytical estimation, we have performed micromagnetic calculations of energy barriers. FEMME implements the elastic band method to find the minimum energy path between to magnetic states. The energy barrier is the difference between the lowest and highest energy on that path [4].

Figure 6 shows the minimum energy path from one magnetic saturated state to the antiparallel saturated state 


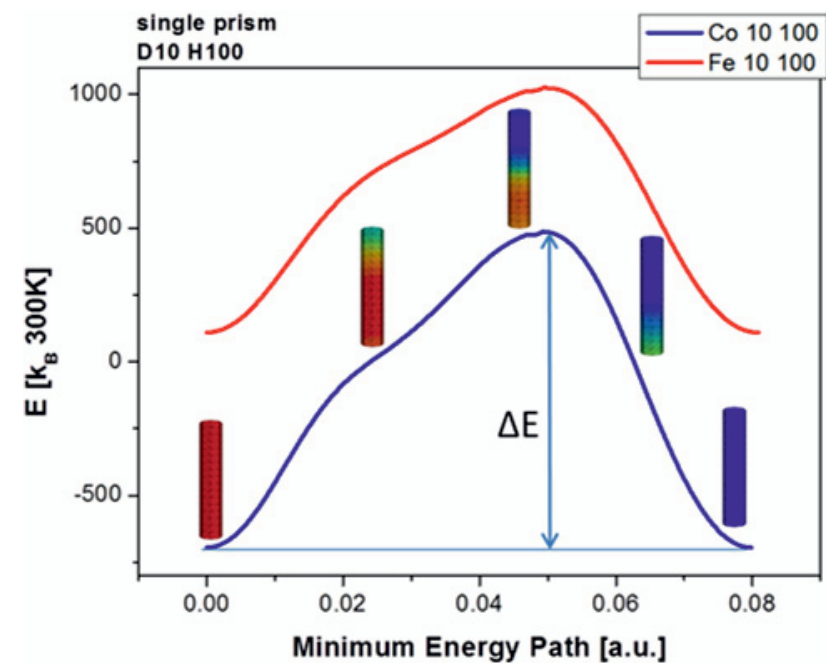

Fig. 6. Minimum energy path of a Co and Fe D10H100 nanorod with magnetic states along the path.

for a Co and Fe D10H100 nanorod with 5 magnetic states along that path.

The simulation results agree with the linear behaviour predicted by the analytical estimate, but not in absolute numbers for both full micromagnetic simulations and simulations without demagnetising field (oHmag, see figure 7). The shape anisotropy gained through the stray field increases thermal stability. But even without stray field effects the simulation results differ from the analytic prediction, because incoherent reversal (curling, buckling and domain wall motion) are energetically more favourable than coherent reversal.

\section{Conclusion}

We introduced an algorithm to generate realistically packed structures consisting of cylindrical nanorods and performed micro magnetic simulations on packed Co nanorod structures. Simulations on the same structure with different calculation methods made it possible to estimate the loss due to incoherent reversal processes and the coercivity gain due to shape anisotropy.

Simulations on single nanorods with varying material parameters have been performed and we predicted the energy density product of a packed structure based on the hysteresis of a single nanorod. As a result, we can give necessary lower limits for $\mathrm{K}_{1}$ and $\mathrm{J}_{\mathrm{s}}$ to reach a particular energy density product.

The results of our energy barrier calculations indicate the nanorods are thermally stable at room temperature, however, the smallest investigated nanorod - D10H50 becomes unstable at $900 \mathrm{~K}$. For nanorods with double volume (D10H100), the thermal stability is a non-issue, because the calculated energy barrier $(2700 \mathrm{~K})$ lies above the melting point.

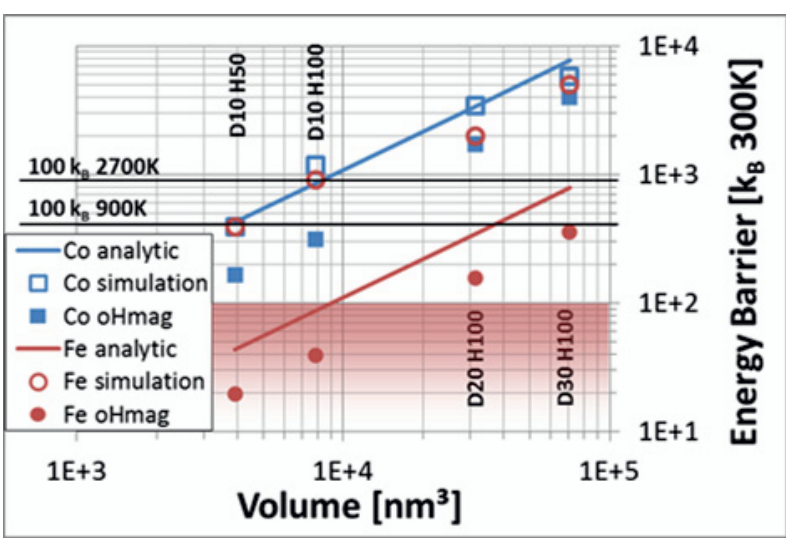

Fig 7. Analytical and simulated energy barriers for Co (square) and $\mathrm{Fe}$ (circle) nanorods with (empty) and without (full) demagnetising field. The thermally unstable range below $100 \mathrm{k}_{\mathrm{E}}$ at room temperature is coloured.

\section{Acknowledgements}

The funding from the European Community's Seventh Framework Programme (FP7-NMP) under grant agreement no. 280670 (REFREEPERMAG) is acknowledged.

\section{References}

1. K. A. Atmane, F. Zighem, Y. Soumare, M. Ibrahim, R. Boubekri, Th. Maurer, J. Margueritat, J.-Y. Piquemal, F. Ott, G. Chaboussant, F. Schoenstein, N. Jouini, G. Viau, J. Solid State Chem. 197, 297-303 (2013)

2. E. Coumans, Bullet Physics Library [Online]. Available: http://www.bulletphysics.com/

3. Th. Schrefl, D. Suess, W. Scholz, H. Forster, V. Tsiantos, J. Fidler, Computational Electromagnetics, 165-181 (Springer Berlin Heidelberg, 2003)

4. R. Dittrich, T. Schrefl, D. Suess, W. Scholz, H. Forster, J. Fidler, J. Magn. Magn. Mater. 250, L12L19 (2002) 